\title{
Improving statistics for hybrid segmentation of high-resolution multichannel images
}

\author{
Elsa D. Angelini ${ }^{\mathrm{a}}$, Celina Imielinska*b, Yinpeng Jin ${ }^{\mathrm{a}}$, Andrew Laine ${ }^{\mathrm{a}}$ \\ ${ }^{a}$ Department of Biomedical Engineering, Fu Foundation School of Engineering and Applied Science, \\ Columbia University, New York, NY \\ ${ }^{\mathrm{b}}$ College of Physicians and Surgeons, Office of Scholarly Resources, Department of Medical \\ Informatics; Department of Computer Science, Columbia University, New York, NY
}

\begin{abstract}
High-resolution multichannel textures are difficult to characterize with simple statistics and the high level of detail makes the selection of a particular contour using classical gradient-based methods not effective. We have developed a hybrid method that combines fuzzy connectedness and Voronoi diagram classification for the segmentation of color and multichannel objects. The multi-step classification process relies on homogeneity measures derived from moment statistics and histogram information. These color features have been optimized to best combine individual channel information in the classification process. The segmentation initialization requires only a set of interior and exterior seed points, minimizing user intervention and the influence of the initialization on the overall quality of the results. The method was tested on volumes from the Visible Human and on brain multi-protocol MRI data sets. The hybrid segmentation produced robust, rapid and finely detailed contours with good visual accuracy. The addition of quantized statistics and color histogram distances as classification features improved the robustness of the method with regards to initialization when compared to our original implementation.
\end{abstract}

Keywords: Hybrid segmentation, Fuzzy connectedness, Voronoi diagram, Color image, multichannel, MRI, Visible Human data.

\section{INTRODUCTION}

There has been much effort in the past two decades for improving segmentation of multichannel images [1-3]. Because of the added complexity of dealing with vector-valued pixels, segmentation of multichannel data is not a completely well defined problem. With single-channel images, object regions are typically modeled as uniform intensity areas and pixel similarity can be measured using Euclidian distance to compare both spatial locations and gray level intensities. However, such approaches can usually not be applied directly to vector-valued pixels. We can identify two general approaches for the segmentation of multichannel images:

(1) The first approach consists of segmenting each channel independently and integrating different contours to get a single segmented object.

(2) The second approach first integrates the vectorial information into a single-value representation of the data and then performs the segmentation on this representation.

The first approach is more intuitive and can fully exploit any strong correlation that exists between individual channels in the edge integration process. On the other hand this method will be limited if edges are defined only as a combination of individual channel information as it is the case with multi-protocol MRI. This setback is analogous to a projection problem. Segmenting individual channel separately can be viewed as projecting the data along separate axis and processing the different projections separately. In this framework, the choice of the projection axis is crucial and the channels decomposition encoding the original data might not be the most appropriate one to use. Moreover the final process of integrating the individual segmented channels can be quite delicate and strongly influence the quality of the segmentation method. For these reasons, the second approach is more robust as it integrates the original vectorial information and not simply the binary edge information derived from individual channels.

This paper presents a new hybrid method that falls in both categories, using homogeneity-based segmentation techniques for fuzzy segmentation and Voronoi diagram classification. These two methods individually fail to produce robust results for minimal initialization when tested independently but achieve great efficiency when combined together as presented earlier [4]. We present in this work new insights for the derivation of a more elaborate homogeneity measure that incorporates color statistics and color histograms in a vectorial fashion for robust segmentation of multi-dimensional data sets.

*ci42@columbia.edu; 701 West $168^{\text {th }}$ street, HHSC201, New York, NY 10032, USA.

Medical Imaging 2002: Image Processing, Milan Sonka, J. Michael Fitzpatrick, 
The general framework of the hybrid segmentation methods is as follows:

(1) A set of seed points are initialized defining the inside and outside regions for the object to segment. Statistics of the two regions are estimated at the seed point locations. The original volume is also quantized with uniform and non-uniform quantization methods described below. This color quantization provides a simplified representation of the vectorial data which is suitable for histogram computation.

(2) A vectorial relative multiscale fuzzy connectedness classifier is applied to extract a good estimate of the object shape using the seed point locations and statistics. The output of this classification is a binary fuzzy mask.

(3) The binary fuzzy mask is used for feature extraction from the original and quantized data for the object to segment. The features extracted include color statistics and quantized color histograms.

(4) An iterative Voronoi diagram classification is performed combining the object features in a homogeneity measure. The output of the Voronoi diagram classification is a binary mask that includes all the objects corresponding to the color features defined by the homogeneity measure.

The general framework is illustrated in the flow chart below:

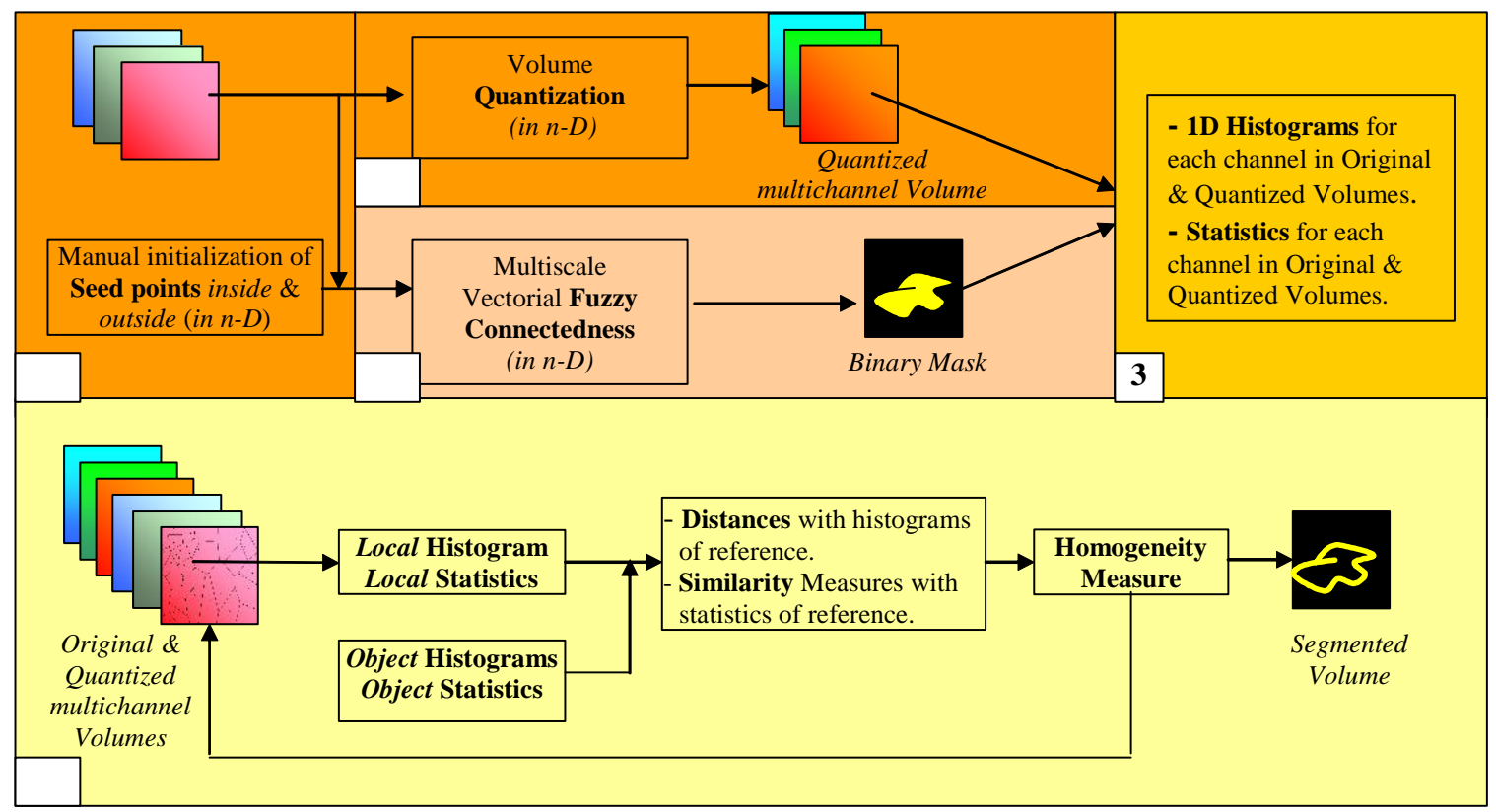

The types of multichannel medical images targeted in this paper include:

(1) Color cryogenic sections from the Visible Human data set. This data set consists of high resolution three dimensional cryogenic sections of a man and a woman cadaver. Segmentation of this data set requires revision of existing traditional methods to accommodate both the colored nature of the data and the unusual high level of spatial detail available $[5,6]$.

(2) Multi-protocol MRI volume of the brain. The data set contains T1-weighted and proton density (PD) MRI images registered for the entire brain of a patient. Tissue characterization via vectorial analysis and classification based using multi-protocol MRI has been investigated in recent works with relative success regarding the automation of the segmentation task [7-10].

(3) Histological image of red blood cells within a capillary vessel. Segmentation of microscopic images is required for cell counting and classification. There is a crucial need to automate this task as the amount of data available and the targeted detail of analysis are exponentially growing in diverse biomedical engineering applications [11, 12].

We detail each component of the method in the following sections, present some results for the different data types and discuss the potentials of the hybrid segmentation method with a focus on possible improvements and new applications. 


\section{INITIALIZATION}

The segmentation is initialized with a set of seed points for computation of local statistics. The seed points are initialized manually on the first slice of the volume with about ten points defining the inside of the object to segment and another ten points for the outside. Local mean and variance statistics are then evaluated within individual channel and covariance statistics estimated between each channel. The covariance measure is only meaningful if the different channels are correlated which is the case for color data but is less meaningful for multi-protocol MRI data.

\section{VECTORIAL RELATIVE MULTISCALE FUZZY CONNECTEDNESS}

Fuzzy connectedness was initially introduced by Udupa [13] and has been successfully used for segmentation of multichannel images in several applications. Fuzzy connectedness is derived from a local vectorial measure called fuzzy affinity. The fuzzy affinity is a measure that assigns to every pair of nearby voxels a strength of local "hanging togetherness". The fuzzy affinity measure has two components called the homogeneity- based affinity and object-based affinity which are devised in a vectorial manner. A global fuzzy relation, called fuzzy connectedness, is then defined on the whole data domain by assigning to every pair of voxels a strength of global hanging togetherness. The strength of hanging togetherness between two voxels (i.e. their connectedness) is defined as the largest strength found over all paths connecting them.

In the multiscale implementation, a scale parameter is assigned to each voxel. This parameter corresponds to the radius of the largest ball centered at the voxel location, within which the vectorial values of all voxels are similar. The similarity measure is based on a maximum difference threshold that we set to $90 \%$ in our implementation. In the multiscale implementation, when determining the affinity between a pair of voxels, all points within a ball associated with both voxels are considered, making the algorithm more robust to noise as reported in [14].

With relative fuzzy connectedness, an object is defined in an image considering the presence of other co-objects. All coobjects of importance present in an image compete together in the process of a voxel assignment as a member of one of them. In this competition process, each pair of voxels in the image has a strength defined relative to each object. The object for which the strength is highest claims the membership. The relative fuzzy connectedness implementation eliminates the need for a threshold to define a voxel membership to a particular object [15]. In this work, for the sake of simplicity, we only defined two objects in the data as the inside the outside of the organ to segment. However, there are no limitations on the number of objects defined in an image, allowing for synchronous segmentation of multiple objects.

\section{EXTRACTION OF OBJECT FEATURE STATISTICS}

Segmentation of the multichannel data is performed with an iterative classification process that tessellates the data domain with Voronoi diagrams and assigns to each Voronoi cell a label corresponding to exterior, interior and boundary cells, based on local homogeneity measures. This iterative scheme was initially described in detail by Chassery et al. [16], applied to gray scale microscopic data in [17] and was extended for segmentation of 2D color images by Imielinska et al. $[4,5,18]$.

In the initial implementation of the hybrid method, only the mean and variance color statistics of the RGB and HSV channels were included in the homogeneity measure.

In this work, we have added new statistics to characterize object color features in terms of texture not limited to color images. To better characterize object texture, we have included color histogram distances in the homogeneity measure. In order to reduce computational complexity and obtain color features with good discrimination power, the original channels are quantized prior to histogram computation. The purpose of channel quantization is to reduce the number of existing colors by aggregating similar colors into a single value. With gray scale images, color similarity is measured with Euclidian distance. Unfortunately, the Euclidian distance as a similarity measure is delicate to extend in $n$-D when working with vectorial representation with strongly correlated channels (as the RGB representation of color images) since close points in the $n$-D color space are not necessarily similar in appearance and do not necessarily correspond to similar tissue type. To address this issue we tested two quantization algorithms and evaluated their performance depending on the space selected for representing the data. These quantization algorithms were: 
(1) Uniform quantization: For each channel, the number of desired color bins is pre-defined, and quantized colors are assigned, equally spaced into a preset number of bins and spanning the original range of values. Within each channel, colors are then linearly mapped to the closest quantized color value.

(2) Non-uniform quantization. The non-uniform quantization method used in this work was introduced by Scharcanski [19]. The strategy of this non-uniform quantization method is to preserve the most occurring $n$-D colors and assign all colors inside a sphere of a preset radius around these predominant colors to the center value. The radius of the sphere is set to accommodate the targeted compression ratio. This radius defines the number of $n$-D colors that encode the final quantized volume but does not control the number of colors in individual channels.

We illustrate in Figure 1 below the different quantization results detained with the two methods for a VH color slice. The original data is coded on 24 bits ( 8 bits per channel). We applied the quantization in both the RGB domain and after transformation in Hue, Saturation, and Value (HSV) representation. The HSV domain provides an uncorrelated representation of the RGB color space. In cylindrical coordinates, the H parameter is encoded as the angle around the axis and sets the tone of the color, the $\mathrm{S}$ parameter is encoded as the distance from the axis and the $\mathrm{V}$ parameter is encoded along the long axis and varies between 0 (blackness) and 1 (whiteness).

For the uniform quantization, the number of color bins was set to [4,4,4] in RGB channels and [18,3,3] in HSV channels as in [20]. For the non-uniform quantization, a representation with 64 colors was targeted for both domains. Because the non-uniform method iteratively aggregates together similar colors, the number of colors obtained at the end of the quantization is usually smaller than the targeted one for images with large homogeneous objects. In the example displayed in Figure 1 the final color maps contained 25 colors for the RGB uniform method, 12 colors for the RGB nonuniform color, 35 colors for the uniform HSV method and 20 for the non-uniform HSV method. We observe in Figure 1 better performance of the non-uniform method to enhance homogeneous regions with a smaller color map. On the other hand, quantization in the HSV domain is very efficient at aggregating colors that correspond to similar texture.

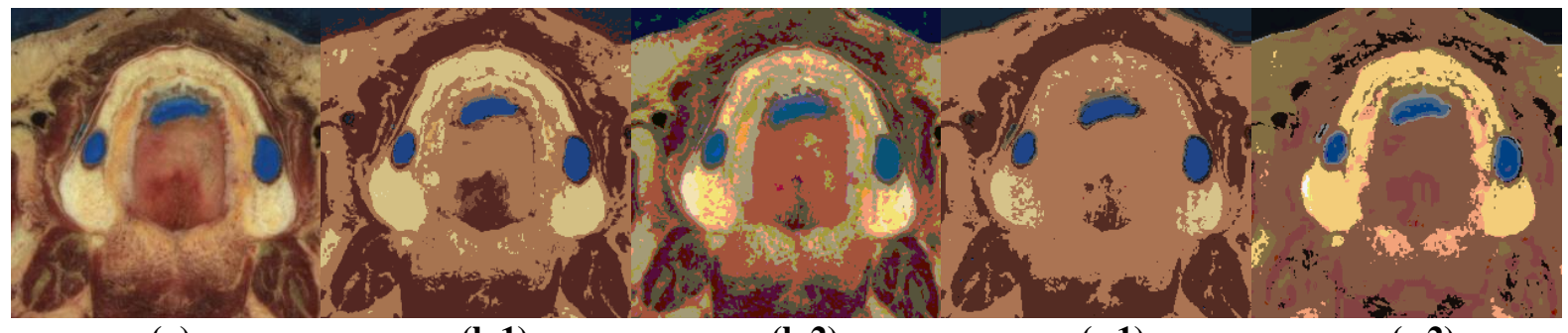

(a)

(b.1)

(b.2)

(c.1)

(c.2)

Fig 1: (a) Original slice from VH data set. (b) Quantization in the RGB domain. (c) Quantization in HSV domain. (b.1-c.1) Non-uniform quantization. Colors are quantized in $\{11,10,10\}$ and $\{16,17,20\}$ bins. (b.2-c.2) Uniform quantization. Colors are quantized in $\{4,4,4\}$ and $\{12,23,10\}$ bins.

These results suggest that high quality quantization can be obtained with non-uniform quantization in the RGB domain or uniform quantization in the HSV domain. This result suggests that uniform quantization is appropriate for representation into non-correlated channels while non-uniform quantization is well suited at enhancing homogeneous areas with high-resolution color representation.

Since the two quantization methods produced images with different properties in terms of enhancement of homogenous areas and preservation of original color aspect, we chose to combine them in the homogeneity measure used by the Voronoi classifier.

After quantization, one-dimensional histograms were computed for the object area defined by the fuzzy mask. Since one histogram is computed for each channel, we extract six histograms in total using the two quantization methods described above.

\section{VORONOI DIAGRAM CLASSIFICATION}

Voronoi diagram tessellation provides a topological division of the space based on nearest neighbor property. In 2D, the Voronoi diagram (VD) for a set $V$ of points is a partition of the Euclidean plane into Voronoi regions of points closer to one point of $V$ than to any other seed point [21]. 
For a set of points $V=\left\{p_{1}, \ldots, p_{n}\right\}$, the Voronoi diagram $V D\left(p_{i}\right)$ associated with the point $p_{i} \in V$ is defined as:

$$
V D\left(p_{i}\right)=\left\{x \in R^{N} \mid d\left(x, p_{i}\right) \leq d\left(x, p_{j}\right), \forall j \neq i, 1 \leq j \leq n\right\},
$$

where $d\left(x, p_{i}\right)$ represents the euclidian distance between points $x$ and $p_{i}$ in $N$ dimensions.

Two Voronoi regions are adjacent if they share a Voronoi edge. The Delaunay triangulation (DT), of $V$ is a dual graph of the Voronoi diagram of $V$, obtained by joining two points whose Voronoi regions are adjacent. An illustration of a Voronoi diagram tessellation and Delaunay triangulation for a given set of points $V$ is provided in Figure 2 below.

The iterative classification process defines Voronoi regions as interior or exterior based on the value of some homogeneity measures evaluated inside the Voronoi region as detailed in [22]. At each step, exterior regions with at least one interior region as a neighbor are labeled boundary regions and split into smaller cells for further classification. This process is iterated until all boundary regions become sufficiently small, based on a preset size threshold. At the end of the iterative process, a subgraph of the Delaunay triangulation is constructed connecting the seed points of the adjacent boundary regions. This graph defines the final outline of the object to segment.

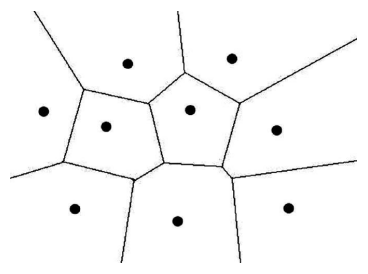

(a)

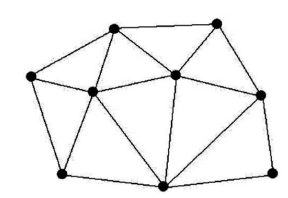

(b)

Figure 2: (a) Voronoi Diagram. (b) Dual Delaunay triangulation.

The definition of the homogeneity measure used by the Voronoi diagram classifier is crucial for the quality of the final segmentation and it has been identified through our previous work as a limiting factor of the hybrid segmentation method [18]. Initial results indicated the efficacy of this method but also suggested that more experiments needed to be carried out to identify classification statistics suited for a wider variety of anatomical structures incorporating more rigorous mathematical descriptions of its textural and structural features. For this reason we tested in this work a new homogeneity measure derived from individual channel statistics and histogram distances. The homogeneity measure is defined as the sum of nine individual distances defined for each channel $i$ as follows:

(1) The percentage error $d_{i}$ between the mean value $m_{i}$ estimated inside the Voronoi cell and the mean value of reference $M_{i}$ of the object to segment:

$$
d_{i}=\left|\frac{m_{i}-M_{i}}{M_{i}}\right|, \quad i=\{1,2,3\} .
$$

(2) The distance $d h_{i}^{R G B}$ between the histogram $h_{i}[k]$ of the colors inside the Voronoi cell and the histogram of reference $H_{i}$ of the object to segment:

$$
d h_{i}=\sum_{k}\left|h_{i}[k]-H_{i}[k]\right|^{r}, \quad i=\{1,2,3\}
$$

Here, $r$ is a non-zero integer that determines the order of norm $L^{r}$. We considered $L^{1}$ and $L^{2}$ norms for the histogram distance terms in our study. Using volumes quantized in RGB and HSV domains we end up with three $d h_{i}^{R G B}$ histogram distances for the RGB quantization and three $d h_{i}^{H S V}$ histogram distances for the HSV quantization.

The homogeneity measure $\Lambda$ is defined as:

$$
\Lambda=\sum_{i=1}^{\text {Channels }}\left(d_{i}<T_{i}\right)+\sum_{i=1}^{\text {Channels }}\left(d h_{i}^{R G B}<T_{i}^{R G B}\right)+\sum_{i=1}^{\text {Channels }}\left(d h_{i}^{H S V}<T_{i}^{H S V}\right) .
$$

The classification rule assigns a Voronoi cell as interior or exterior depending on a threshold $T$ :

If $\Lambda>T$ then the cell is classified as interior

If $\Lambda \leq T$ then the cell is classified as exterior. 
For the color differences, the $T_{i}$ thresholds are defined as:

$$
T_{i}=2 * \Sigma_{i} / M_{i},
$$

where $M_{i}$ and $\Sigma_{i}$ correspond to the mean and standard deviation of the object color in the channel $i$. This model assumes a Gaussian distribution of the color with a characteristic interval of $2 \Sigma_{i}$ radius. This assumption is reasonable in the original color space for all high-resolution images and also in quantized domains, in that the quantization preserves the smoothness of the color distribution within homogeneous areas.

For histogram distances, the choice of both the distance and the threshold is quite delicate and has been the focus of active research and discussion in the field of color image indexing [20, 23-26]. The main challenge in using histogram distances as a similarity measure relies in the fact that (1) small differences can be amplified and (2) distance value doesn't reflect the degree of perceptual difference between two images. Color quantization will minimize these effects. To further control the range of distances measured in the homogeneity function, we normalized the histograms to 1 , so that the maximum value of the distance between any two is equal to 2 . As the order of the norm $L^{r}$ increases, distances between histogram bins will decrease since we are working with numbers smaller than one. This means that small differences are less amplified at the cost of less discriminatory power. As the number of bins in the histogram increase, the distance value at each bin decreases, as you represent the same object with more colors. The rate of decrease of the distance with the number $N$ of bins in the histogram is of the order of $N^{r}$ where $r$ is the norm order. These observations motivated the choice of the following threshold for the histogram distance with a $L^{r}$ norm:

$$
T_{i}^{R G B / H S V}=N \times(2 \tau)^{r},
$$

where $\tau$ is an error threshold that we set between $10 \%$ and $40 \%$ in our implementation. With high quantization rates as applied here, we observed that the $L^{2}$ norm was an adequate choice with satisfactory selectivity.

The global threshold value $T$ was set to the total number of tests such that all of them were verified for a cell to be classified as interior.

\section{TESTING ON MULTICHANNEL DATA}

We present in this section results for the following multichannel data:

(1) Multi-modality MRI of a brain including T1-weighted and positron density (PD) images,

(2) Cryogenic sections from the Visible Human data set including the maxilla and the mandible bones,

(3) Cryogenic sections from the Visible Human data set including adipose fat tissue,

(4) Histological image of red blood cells within a capillary.

For the quantization of color data sets, we decided to include both a fine color-preserving quantization performed in the RGB domain with the non-uniform method and a uniform quantization performed in the HSV domain. Quantization was performed in the RGB color space into 64 colors (2 bits per channel) and in the HSV domain into [18,3,3] bins for the Hue, Saturation and Value dimensions. Following a similar approach as in [20] we divide the Hue circle into 18 bins at $20^{\circ}$ steps which leads to an efficient coding of the three primary colors and their three complementary colors on three sub-divisions each. Saturation and value are coded on three bins each to achieve a good selectivity in visual perception. For the MRI data, the two channels corresponding to T2-weighted and PD are not strongly correlated since these two protocols enhance different tissue types within the brain. Moreover, the T2 channel has a very low contrast between the white and gray matter. For these two reasons, the uniform quantization method performed poorly at enhancing the different tissues corresponding to the gray matter, the white matter, and the cerebrospinal fluid (CSF). We therefore only applied non-uniform quantization in the original domain with 4 bits per channel.

Quantization results for the five types of data are illustrated in Figure 3 below. 


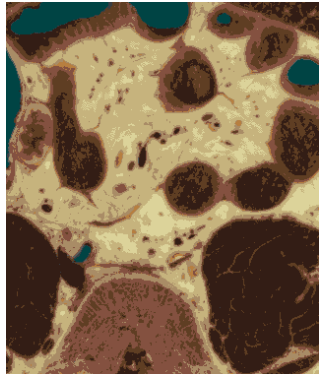

(a)
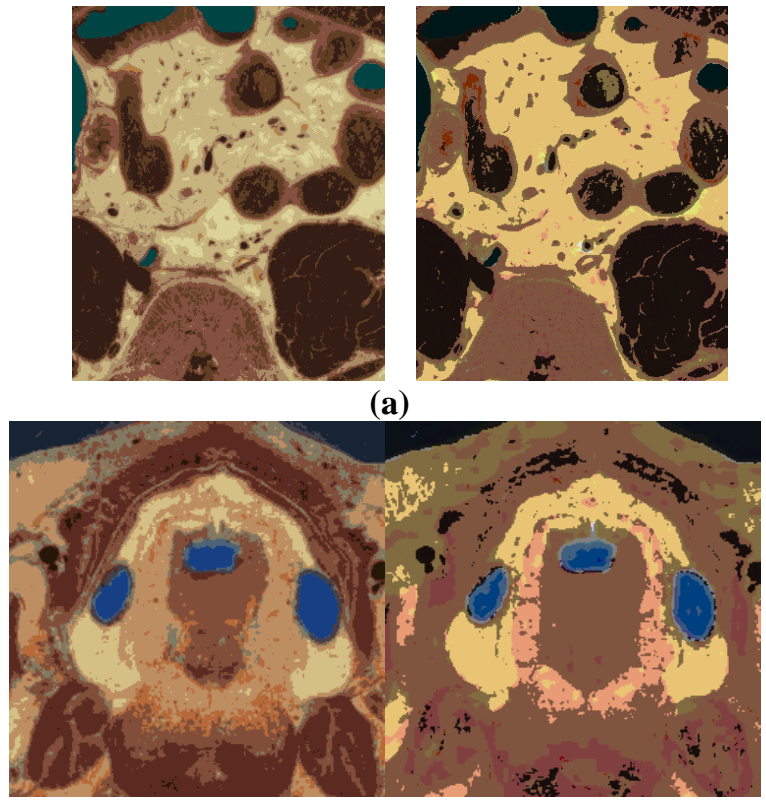

(c)
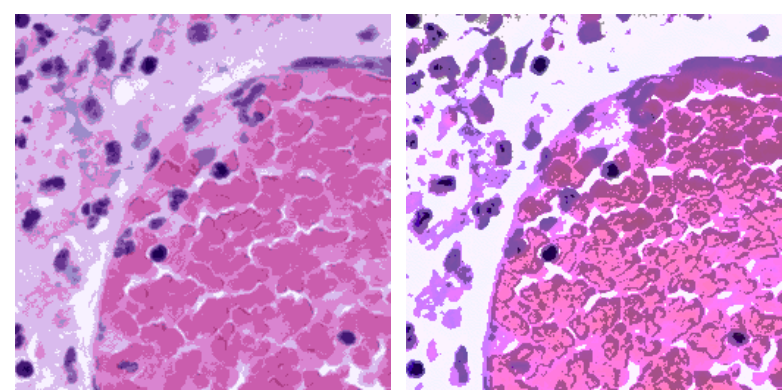

(b)
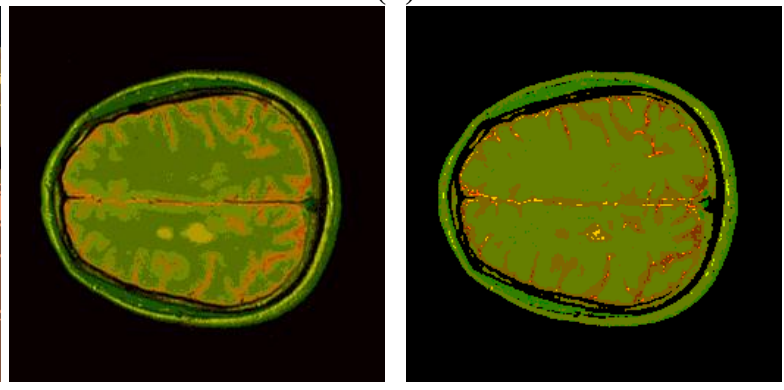

(d)

Fig 3: Uniform and non-uniform color quantization. (a) Slice from the VH data set with fat tissue. (b) Microscopic image of red blood cell. (c) 3D HV volume with maxilla (upper jaw) bone. (d) Two-channel brain MRI.

The purpose of the color quantization is to provide histograms that can characterize the color distribution for the object to segment in a simplified manner. As can be observed in Figure 3, the two quantization methods provided results with variable selectivity, both enhancing different features for the targeted objects.

Regarding the fuzzy connectedness, we observe in the examples displayed in Figure 4 below that the method, applied in a simplified manner with a two-object setting, provides a partial segmentation of the object of interest with fine contour details.
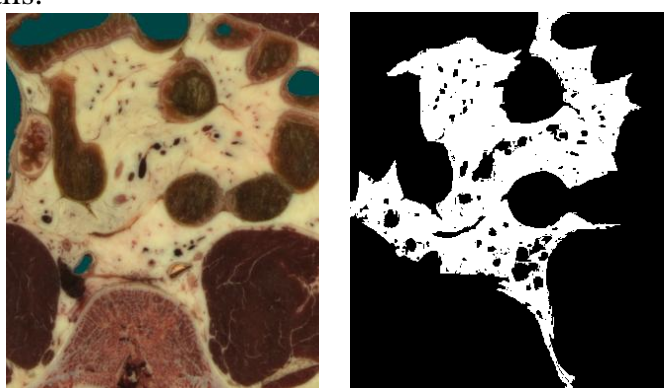

(a)

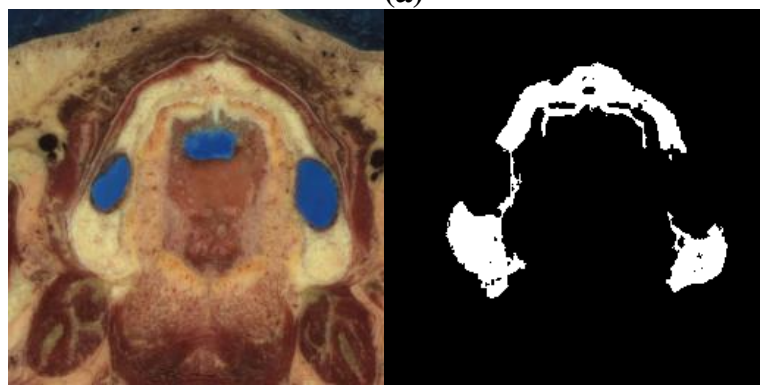

(c)

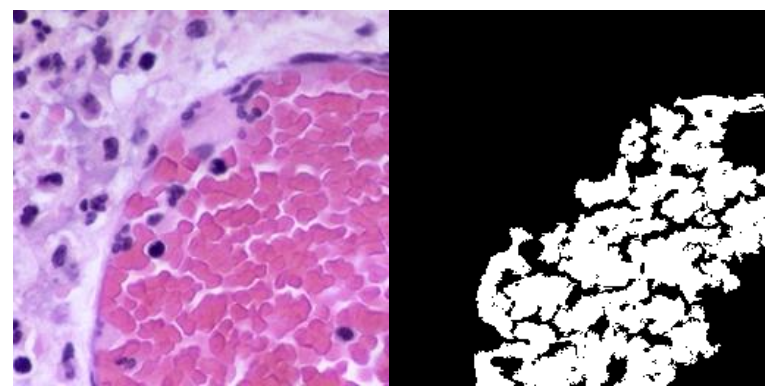

(b)

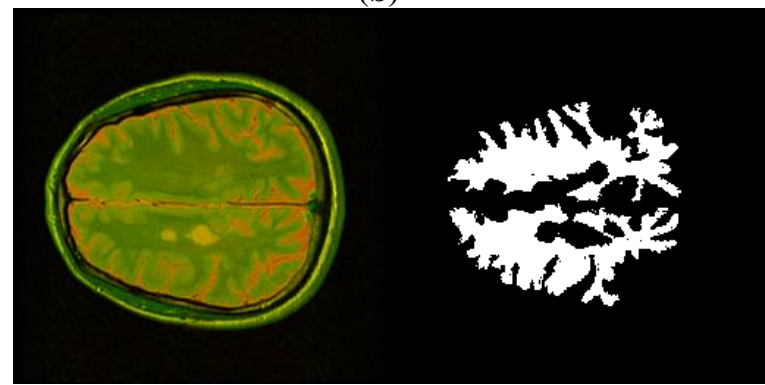

(d)

Fig 4: Binary masks extracted by the vectorial multiscale fuzzy connectedness classification. (a) VH slice with fat tissue. (b) Microscopic image of red blood cell. (c) VH volume with maxilla (upper jaw) bone. (d) Two-channel brain MRI. 
The binary masks derived from the fuzzy connectedness classification defined the sub-volume used for the estimation of the color features of the object to segment. The color features included:

(1) The global mean value and standard deviation of the object colors in the original and the quantized volumes,

(2) The object color histograms for each channel of the quantized volumes.

The color and histogram information is incorporated into the homogeneity measure using percentage error and $L^{2}$ norm distances between histograms. We illustrate in Figure 5, below, for the maxilla bone example, the characteristics of the color histogram components and the corresponding distance terms in the homogeneity function.

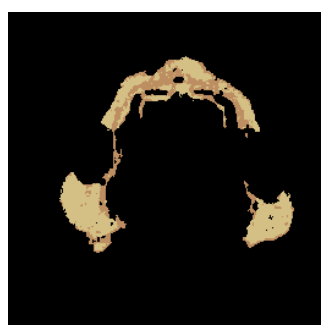

(a1)

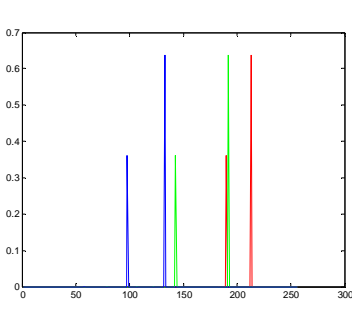

(a2)

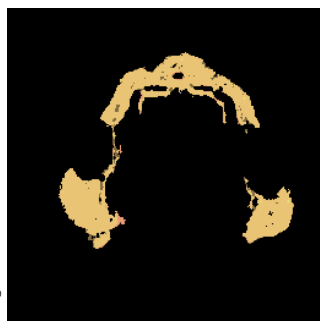

(b1)

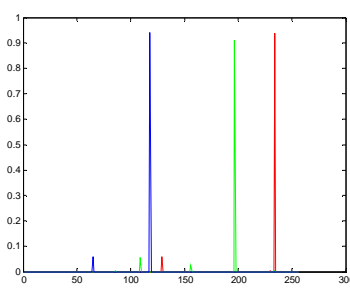

(b2)
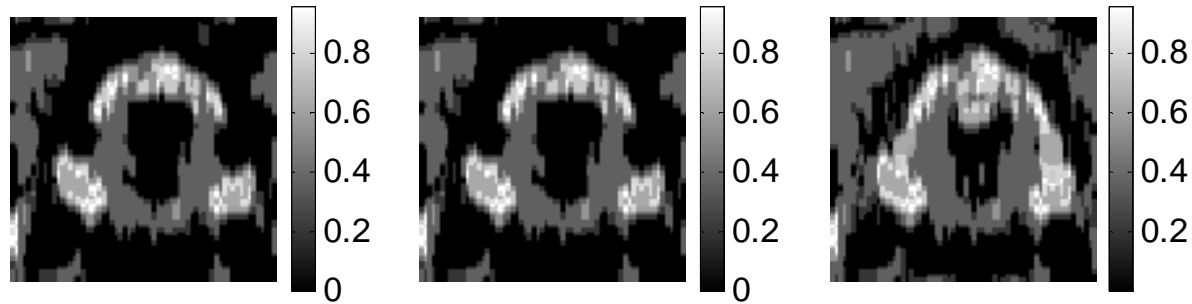

(c)
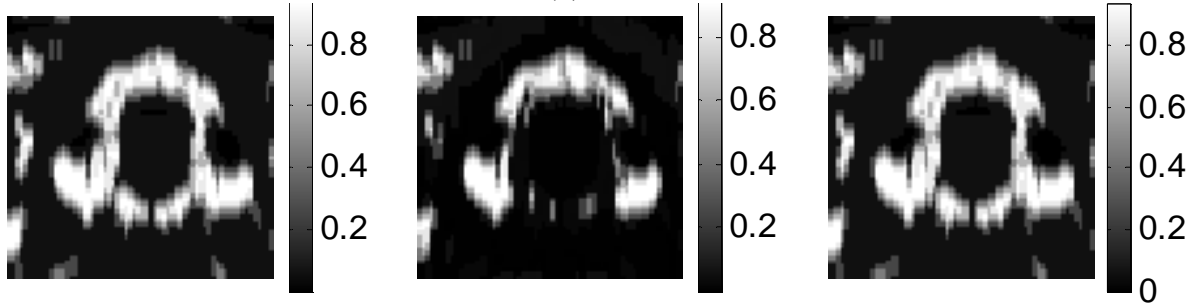

(d)

Fig 5: Example of homogeneity measure components for VH slice with maxilla (upper jaw) bone. (a1) Fuzzy mask applied to volume quantized in RGB domain. (a2) Object area histogram in Red, Green and Blue channels. (b1) Fuzzy mask applied to volume quantized in HSV domain. (b2) Object area histogram in Red, Green and Blue channels. (c) Histogram distance maps using non-uniformly quantized volume (RGB domain). (d) Histogram distance maps using uniformly quantized volume (HSV domain). Distance maps are computed for the Red, Green and Blue channels. The color scale was inverted for display purposes so that higher (white) values correspond to lower distances.

The Voronoi classification algorithm was run until the neighbor cells contained less than ten pixels and a binary mask of the interior cell was extracted. Results of the Voronoi diagram classification are displayed in Figure 6 below, where the final binary mask is overlaid with the original data to better appreciate the quality of the segmentation for the specific targeted tissues. 

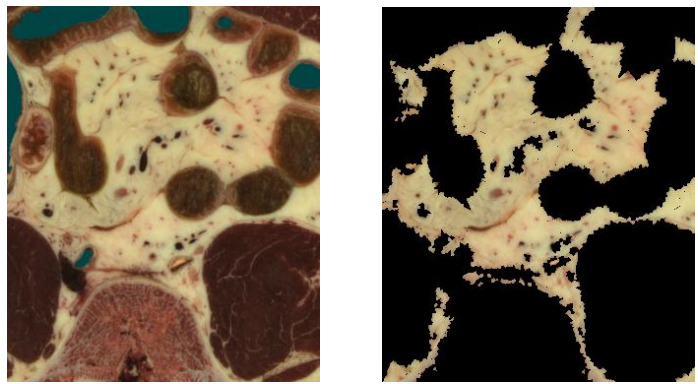

(a)
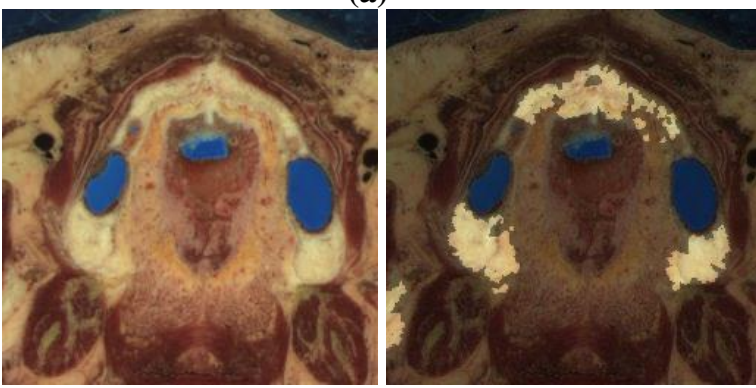
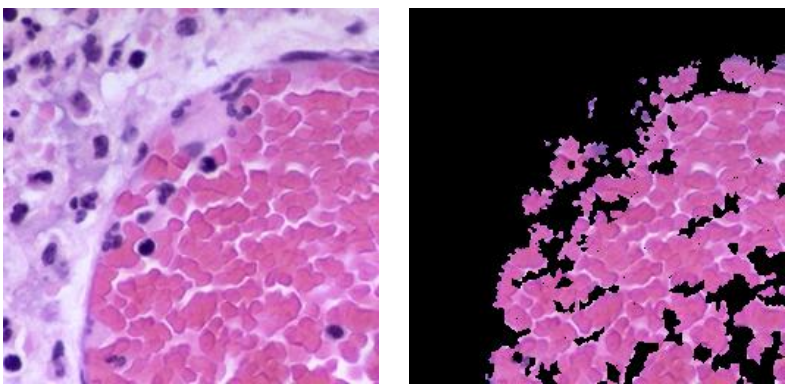

(b)
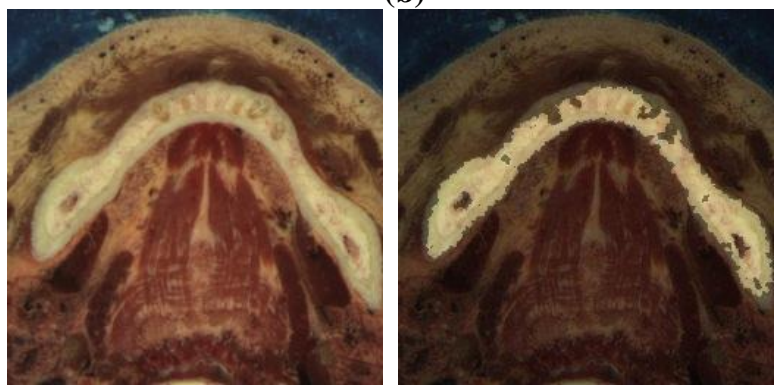

(c)
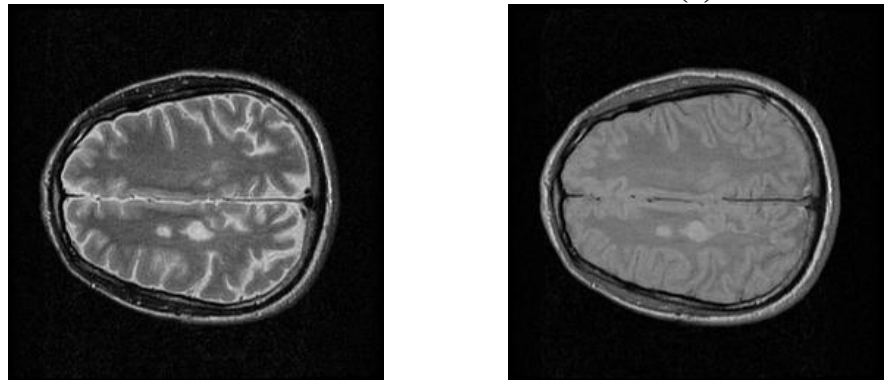

(d)

Fig 6: Binary masks of final segmentation overlaid on original data. (a) Slice from the VH data set with segmentation of adipose fat tissue. (b) Microscopic slice with segmentation of red blood cell within a capillary. (c) VH volume with segmentation of the maxilla (upper jaw) and mandible (lower jaw) bones. (d) Two-channel brain MRI with segmentation of the white matter.

For the five examples presented here, the hybrid segmentation method was observed to be very efficient at extracting the contours of the organs of interest with fine details. The diversity of image type and tissue appearance presented in this series of tests shows the potential of the hybrid method to handle:

(1) Objects with multiple parts such as the white matter, the ref blood cells and the maxilla bone,

(2) Objects with complex shapes such as the white matter,

(3) Objects with fuzzy boundaries such as the jaw bones,

(4) Objects with complex textures such as the fat tissue and the red blood cells.

\section{DISCUSSION}

The hybrid method combined vectorial information in a hierarchical manner letting the individual channels to either fuse or compete for the characterization of the object to segment. Examples presented in the Results Section showed the diversity of segmentation tasks that this type of hybrid algorithm can handle within a simple common framework. The fuzzy connectedness component was able to provide very accurate but fragmented maps of the object to segment. However, from these maps, precise statistics and histogram could then be extracted to characterize the texture of each object in vectorial dimension. The final segmentation was obtained with a Voronoi classification based on a new homogeneity function that incorporates the textural features to combine individual channel information. The Voronoi tessellation allowed for multi-part segmentation, as illustrated in each of the five examples and relied only on the 
definition of an interior, letting the outside be anything that does not correspond to the interior definition. The Voronoi tessellation offered a very flexible partitioning of the space into local neighbor segments whose size depends on the local homogeneity of the data. As the classification process evolves from a global to a local segmentation of the space, only areas that include part of the organ to segment are further processed in the next iteration. This property reduces the complexity of the algorithm while refining the localization of edges inside smaller neighbor Voronoi cells. The adaptive topology of the cell performs a portioning of the space into small elements that can handle any complex curvatures along the organ contours without global constraints as with deformable model. Furthermore, the iterative splitting of neighbor cells let the classification process propagates within adjacent cells and correct for initial misclassification of some large Voronoi cells by smaller ones.

As this work presents preliminary results, further testing will be carried out to provide quantitative validation of the method. Future clinical applications of this method will address the challenging segmentation task of fat tissue from multi-protocol MRI images for quantitative analysis of body composition. Further refinement of the method will focus on incorporating edge information in the homogeneity function using $n$-D vectorial gradient operators as presented by Cumani in [27]. Another direction for improving the method will also experiment with statistics derived from additional analysis domains such as time-frequency and wavelet expansions for better texture characterization.

\section{CONCLUSION}

We have presented in this paper new insights for the segmentation of multichannel medical images with a hybrid method that combines fuzzy connectedness and Voronoi diagram classification. The hybrid method offers more robustness than simple boundary or region-based algorithms for the segmentation of high-resolution multichannel medical images. The field of application of segmentation methods for vectorial medical images is becoming very broad with the introduction of multi-protocol patient screening $[8,9,28,29]$, the evolving field of multispectral and optical imaging, the need for automated processing tool for microscopic data in cellular and genetic research and the advancements in endoscopic screening. We can also mention the original work of Muraki et al. [30, 31] in coloring multichannel data using Visible Human color textures mapped to multi-protocol MRI brain data.

The components of the software were implemented with itk libraries, a freeware Segmentation and Registration Toolkit developed by the Insight Consortium (http://www.itk.org).

\section{ACKNOWLEDGEMENTS}

This work was supported in part by National Library of Medicine contract on the "VHP Segmentation and Registration Toolkit" - NLM99-103/DJH.

\section{REFERENCES}

[1] P. M. Djuric and J.-K. Fwu, "On the detection of edges in vector images," IEEE Transactions on Image Processing, vol. 6, pp. 1595-1601, 1997.

[2] S. Wesolkowski, M. E. Jernigan, and R. D. Dony, "Comparison of color image edge detectors in multiple color spaces," Proceedings of International Conference on Image Processing, Vancouver, BC, Canada, pp. 796 - 799, 2000.

[3] A. Koschan, "A comparative study on color edge detection," Proceedings of 2nd Asian Conference on Computer Vision, Singapore, pp. 574-578, 1995.

[4] C. Imielinska, D. Metaxas, J. Udupa, Y. Jin, and T. Chen, "Hybrid segmentation of anatomical data," Proceedings of Medical Image Computing and Computer Assisted Intervention - MICCAI, Utrecht, The Netherlands, pp. 1048-1057, 2001.

[5] C. Imielinska, M. Downes, S. Hosakere, A. Khan, and W. Yuan, "2D segmentation of color anatomical data," Proceedings of First User Conference of the National Library of Medicine's Visible Human Project, Bethesda, MD, USA, 1996.

[6] A. Pommert, K. H. Hohne, B. Pfesser, E. Richter, M. Riemer, T. Schiemann, R. Schubert, U. Schumacher, and U. Tiede, "Creating a high-resolution spatial/symbolic model of the inner organs based on the Visible Human," Medical Image Analysis, vol. 5, pp. 221-228, 2001.

[7] M. Vannier, T. Pilgram, C. Speidel, L. Neumann, D. Rickman, and L. Schertz, "Validation of magnetic resonance imaging (MRI) multispectral tissue classification," Computerized Medical Imaging and Graphics, vol. 15, pp. 217-223, 1991. 
[8] H. S. Choi, D. R. Haynor, and Y. Kim, "Partial volume tissue classification of multichannel magnetic resonance images-a mixel model," IEEE Transactions on Medical Imaging, vol. 10, pp. 395 - 407, 1991.

[9] R. Sammouda, N. Niki, and H. Nishitani, "Multichannel segmentation of magnetic resonance cerebral images based on neural networks," Proceedings of International Conference on Image Processing, Washington, DC, USA, pp. 484 - 487, 1995.

[10] Y. Sato, C. Westin, A. Bhalerao, S. Nakajima, N. Shiraga, S. Tamura, and R. Kikinis, "Tissue classification based on 3D local intensity structures for volume rendering," IEEE Transactions on Visualization and Computer Graphics, vol. 6, pp. 160 - 180, 2000.

[11] C. D. Ruberto, A. Dempster, S. Khan, and B. Jarra, "Analysis of infected blood cell images using morphological operators," Image and Vision Computing, vol. 20, pp. 133-146, 2002.

[12] J. M. Chassery, "Microscopic Imaging," Proceedings of Annual International Conference of the IEEE Engineering in Medicine and Biology Society, Orlando, FL, USA, pp. 1113 - 1114, 1991.

[13] J. K. Udupa and S. Samarasekera, "Fuzzy connectedness and object definition: Theory, algorithms and applications in image segmentation," University of Pennsylvania, Philadelphia MIPG 209, 1995.

[14] P. K. Saha, J. K. Udupa, and D. Odhner, "Scale-based fuzzy connected image segmentation: theory, algorithms, and validation," Computer Vision and Image Understanding, vol. 77, pp. 145-174, 2000.

[15] P. K. Saha and J. K. Udupa, "Relative fuzzy connectedness among multiple objects: Theory, algorithms, and applications in image segmentation," Computer Vision and Image Understanding, pp. 42-56, 2001.

[16] M. Melkemi and J. M. Chassery, "Edge-region segmentation process based on generalized Voronoi diagram representation," Proceedings of International Conference on Pattern Recognition, The Hague, Netherlands, pp. 323 - 326, 1992.

[17] E. Bertin, F. Parazza, and J. M. Chassery, "Segmentation and measurement based on 3D Voronoi Diagram: application to confocal microscopy," Computerized Medical Imaging and Graphics, vol. 17, pp. 175-182, 1993.

[18] C. Imielinska, D. Metaxas, J. Udupa, Y. Jin, and T. Cheng, "Hybrid segmentation of the Visible Human data," Proceedings of Third Visible Human Project Conference, Bethesda, MD, USA, 2000.

[19] J. Scharcanski, H. C. Shen, and A. P. A. d. Silva, "Colour quantisation for colour texture analysis," Proceedings of Computers and Digital Techniques, pp. 109 - 114, 1993.

[20] J. R. Smith, "Integrated Spatial and Feature Image Systems: Retrieval, Analysis and Compression," in Electrical Engineering Department. New York: Columbia University, 1997, pp. 176.

[21] F. P. Preparata and M. I. Shamos, Computational Geometry. New York: Springer, 1985.

[22] C. Imielinska, M. Downes, and W. Yuan, "Semi-automated color segmentation of anatomical tissue," Computerized Medical Imaging and Graphics, vol. 24, pp. 173-180, 2000.

[23] C. L. Novak and S. Shafer, "Estimating Scene Properties from Color Histograms," Computer Science Department, Carnegie Mellon University, Technical Report CMU-CS-92-212, 1992.

[24] M. Stricker and M. Swain, "The capacity of color histogram indexing," Proceedings of IEEE Conference on Computer Vision and Pattern Recognition, Seattle, WA, pp. 704 - 708, 1994.

[25] M. J. Jones and J. M. Rehg, "Statistical color models with application to skin detection," Proceedings of IEEE Conference on Computer Vision and Pattern Recognition, pp. 274-278, 1999.

[26] M. Stricker and M. Orengo, "Similarity of color images," Proceedings of SPIE Conference on Storage and Retrieval for Image and Video Databases, 1995.

[27] A. Cumani, "Edge detection in multispectral images," Istituto Elettrotecnico Nazionale "Galileo Ferraris" R.T. 373, 1989.

[28] P. Scheunders, "Multivalued image segmentation based on first fundamental form," Proceedings of International Conference on Image Analysis and Processing, Palermo, Italy, 2001.

[29] H. H. Pien and J. M. Gauch, "Variational segmentation of multi-channel MRI images," Proceedings of IEEE International Conference Image Processing, Austin, TX, USA, pp. 508 - 512, 1994.

[30] S. Muraki, T. Nakai, and Y. Kita, "Basic research for coloring multichannel MRI data," Proceedings of Visualization, Salt Lake City, UT, USA, pp. 197-194, 2000.

[31] S. Muraki, T. Nakai, Y. Kita, and K. Tsuda, "An attempt for coloring multichannel MR imaging data," IEEE Transactions on Visualization and Computer Graphics, vol. 7, pp. 265 - 274, 2001. 